\section{Uneven distribution of NG2 cells in the rat cerebellar vermis and changes in aging}

\section{S. Lomoio, ${ }^{1}$ D. Necchi, ${ }^{2}$ E. Scherini ${ }^{1}$}

'Dipartimento di Biologia e Biotecnologie Lazzaro Spallanzani, Università di Pavia; 2Dipartimento di Scienze del Farmaco, Università di Pavia, Italy

\begin{abstract}
We describe by NG2 (neuron-glia chondroitin sulphate proteoglycan 2) immunocytochemistry an uneven distribution of NG2 glial cells in the rat cerebellum, being them more represented in the central lobules of the cerebellar vermis, belonging to the cerebrocerebellum. The cerebellar distribution of NG2 cells changes in aging rats, in which the area where the cells appear to be densely scattered throughout all cerebellar layers involves also more rostral and caudal lobules. In addition, in aging rats, in the most rostral and caudal lobules belonging to the spinocerebellum, punctate reaction product is present at the apical pole of Purkinje cells, i.e. in the area where the majority of synapses between olivary climbing fibers and Purkinje cells occur. Data suggest that the different distribution of NG2 cells is correlated to differences in physiology among cerebellar areas and reflects changes during aging.
\end{abstract}

\section{Introduction}

NG2 cells (neuron-glia chondroitin sulphate proteoglycan 2), also referred to as synantocytes,${ }^{1}$ polydendrocytes,${ }^{2} \beta$-astrocytes,${ }^{3}$ oligodendrocyte precursor cells (OPC) ${ }^{4}$ have been recognized as the fourth type of glial cells in the mammalian central nervous system, representing about $5-8 \%$ of the glial cell population. Morphologically, at light microscopy they resemble astrocytes, though their cell body is less rounded and cytoplasmic processes slender. At electron microscopy, NG2 cells show flattened nucleus with decondensed chromatin, well-represented cytoplasmic orga nelles and, where opposed to neurons, focal membrane densities. ${ }^{5}$ Opposite to astrocytes, NG2 cells do not express GFAP nor S100 protein, but express the NG2 chondroitin sulphate proteoglycan, a membrane protein with a large extracellular domain, whose function is still obscure. In the central nervous system, NG2 cells can be found in both gray and white matter and have been proposed as resident oligo- dendrocyte and astrocyte precursors. Actually, their number increases in the vicinity of demyelization foci in some neuropathologies and after wound insult. ${ }^{6-8}$ On the other hand, NG2 cells do not express glutamate transporters, but have calcium-permeable AMPA receptors, that cluster in apposition to nerve terminals, forming conventional synapses. ${ }^{9-11}$ This feature suggests that these cells must have a more complex function than to be a simple reservoir of oligodendrocytes or astrocytes. The cerebellum, which has a pivotal role in motor control, timing and learning, has been relatively neglected in studies concerning aging. In elderly people, movement and stability control are less efficient, with increasing fall risk and serious consequences and social costs. Here, we describe that NG2 cells are not evenly distributed in the rat cerebellum and that their distribution and morphology undergo changes during aging.

\section{Materials and Methods}

Sprague Dawley rats, aged 4 (3 animals) and 28 months (3 animals), purchased from Harlan Nossan (Correzzana, Italy) and maintained in standard conditions (12 h light/dark cycle, $23^{\circ} \mathrm{C}$ temperature, food and water ad libitum) for few days were used. Experiments were performed in accordance with the Italian and European Community law for the use of experimental animals and were approved by a local bioethical committee.

Animals were deeply anesthetized with chloral hydrate $(0.35 \mu \mathrm{g} / \mathrm{g}$ body weight $)$ and perfused transcardially with $4 \%$ paraformaldehyde in PBS. Cerebella were excised, postfixed in the same fixative for $4 \mathrm{~h}$ and cryoprotected in $30 \%$ sucrose overnight. The cerebella were sectioned serially into $25 \mu \mathrm{m}$-thick sagittal sections. Two out of every 10 sections were processed for immunocytochemistry for NG2 chondroitin sulphate proteoglycan as follows. Briefly, after treatment with $3 \% \mathrm{H}_{2} \mathrm{O}_{2}$ in $10 \%$ methanol in PBS and with $10 \%$ normal serum in PBS containing $0.1 \% \mathrm{NaN}_{3}$, the sections were incubated overnight with 1:200 rabbit polyclonal antibody against NG2 chondroitin sulphate proteoglycan (Chemicon, Billera, MA, USA). Afterwards the sections were incubated for $90 \mathrm{~min}$ in anti-rabbit biotinylated IgG, followed by streptavidin-HRP complex (Vectastain Elite kit, Vector, CA, USA). The complex was revealed by 3 ', 5'-diaminobenzidine tetrahydrochloride. Some adjacent sections from 28-month-old rats were processed for calbindin immunocytochemistry (with 1:5000 rabbit polyclonal antibody against calbindin, Swant, $\mathrm{CH}$ ) to check for the integrity of the cytoarchitecture. For the reaction control,
Correspondence: Elda Scherini, Dipartimento di Biologia e Biotecnologie Lazzaro Spallanzani, Laboratorio di Biologia Cellulare e Neurobiologia, Università di Pavia, via Ferrata 9, 27100 Pavia, Italy.

Tel. +39.0382.986320 - Fax: +39.0382.986325.

E-mail: scherini@unipv.it

Key words: cerebellum, aging, NG2 glia.

Contributions: all authors contributed equally to the work.

Conflict of interests: the authors declare no conflict of interests.

Received for publication: 15 February 2012. Accepted for publication: 24 April 2012.

This work is licensed under a Creative Commons Attribution NonCommercial 3.0 License (CC BYNC 3.0).

CC Copyright S. Lomoio et al., 2012

Licensee PAGEPress, Italy

European Journal of Histochemistry 2012; 56:e27 doi:10.4081/ejh.2012.e27

some sections were incubated with normal rabbit serum instead of the primary antibodies. To quantify the changes in the cerebellar area in which NG2 glia was densely scattered, sections were photographed at low magnification and a morphometric analysis was performed using NIH Image J software. The total section area and the area in which NG2 cells were densely represented (relative area) were measured by outlining the contours. Since the total area of cerebellar sections can vary with age and among animals of the same group, the ratio between the relative and total areas was calculated to standardize data. Data were statistically analyzed by Kruskal-Wallis test.

\section{Results and Discussion}

In the cerebellar vermis of 4-month-old rats, NG2 cells appear to be unevenly distributed. In lobules VI to VIII according to Larsell ${ }^{12}$ NG2 cells are uniformly and densely scattered in all cerebellar layers, including the white matter (WM); their long, slender cytoplasmic processes extend to form a spider web-like structure throughout all the cerebellar cortex (Figure 1a). On the contrary, in the more rostral and caudal lobules (I-VI and IX-X), NG2 cells are less represented. Only sporadic cells may be observed in the WM and the granule cell layer (IGL), the majority of cells lying in the molecular layer (ML). In addition, they appear less intensely stained than in the more central lobules (Figure 2a). In control reaction sections 
no immunostaining is present (Figure 1c). The uneven distribution of NG2 cells may reflect differences in fiber connections among lobules. In fact, in mammals the central part of cerebellar vermis receives its main input from the cerebral cortex and is therefore called cerebrocerebellum. The anterior and posterior parts receive afferents primarily from the spinal cord and are therefore termed spinocerebellum. With regard to efferent connections, the cerebrocerebellum acts on the cerebral cortex and the spinocerebellum influences the spinal cord. In particular, the cerebrocerebellum receives information, via the corticopontine tract, about movements that are being planned and about commands that are sent out
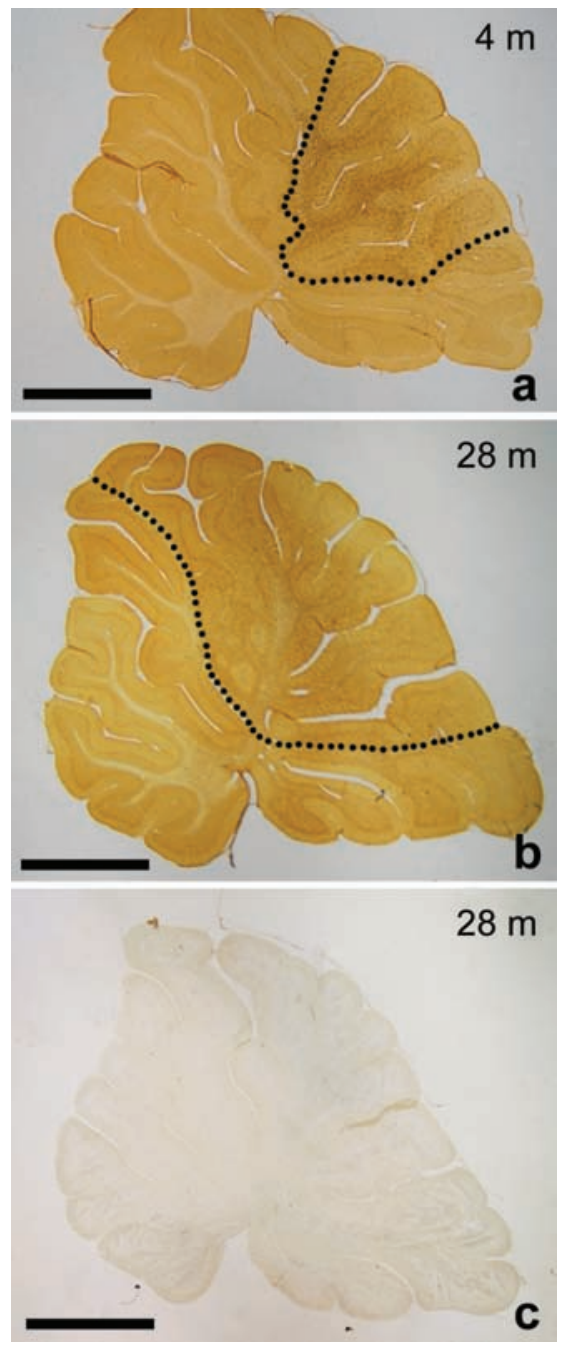

Figure 1. a, b) Representative sections of the cerebellar vermis of 4- and 28-month-old rats showing the distribution of NG2 cells. The cerebellar area in which NG2 cells are uniformly and densely scattered (dotted line) is enlarged in aging animals; C) a section from a 28-month-old animal incubated with normal rabbit serum instead of the primary antibody. No immunoreaction is present. Scale bar: $2 \mathrm{~mm}$. from the motor cortex. In response, it can modulate the activity of the motor cortex so that movements are performed accurately and smoothly. The high density of NG2 cells in the central lobules of the cerebellar vermis in the rat may therefore be due to a higher functional complexity of the cerebrocerebellum, which in turn requires a higher modulation from glial cells. As already mentioned, NG2 cells show conventional synapses with neurons. ${ }^{13}$ The functional significance of this rapid communication between neurons and NG2 cells is still obscure, but it has been suggested that through synapses NG2 cells can monitor neuronal activity and act consequently, maybe by releasing neurotrophic substances. ${ }^{14}$

The distribution of NG2 cells in the cerebellar vermis of 28-month-old rats is similar to that observed in 4-month-old animals. However, the area in which NG2 cells are more represented and uniformly scattered (relative area) appears to be enlarged, involving also the lobule V and part of the lobule IX (Figure
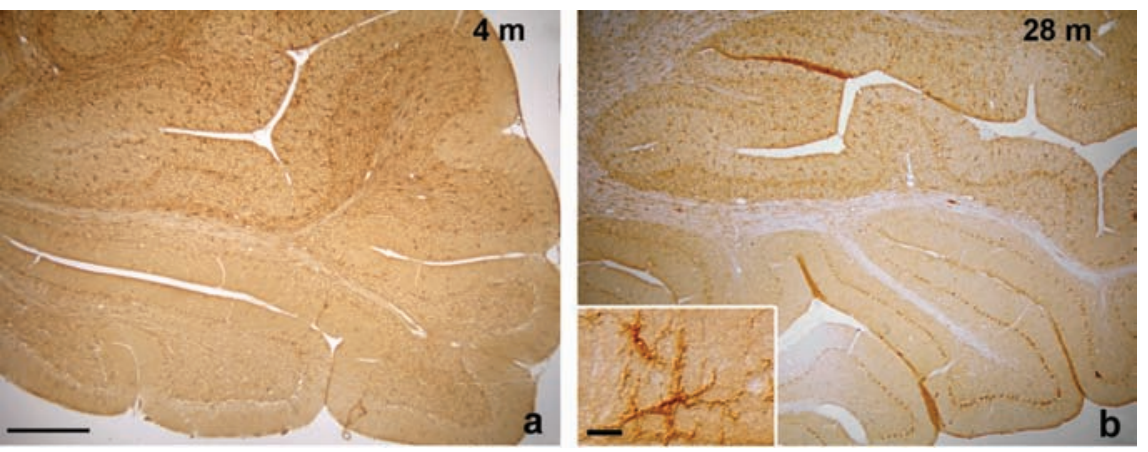

Figure 2. In both 4- and 28-old rats, in the more rostral and caudal lobules of the cerebellum, NG2 cells are only sporadically present, mainly in the molecular layer. Inset in b: in the molecular layer of both 4-and 28-month-old animals, immunostained cells show irregular soma and long, slender processes, typical of NG2 glia. Scale bar a,b: $400 \mu \mathrm{m}$; inset $20 \mu \mathrm{m}$.
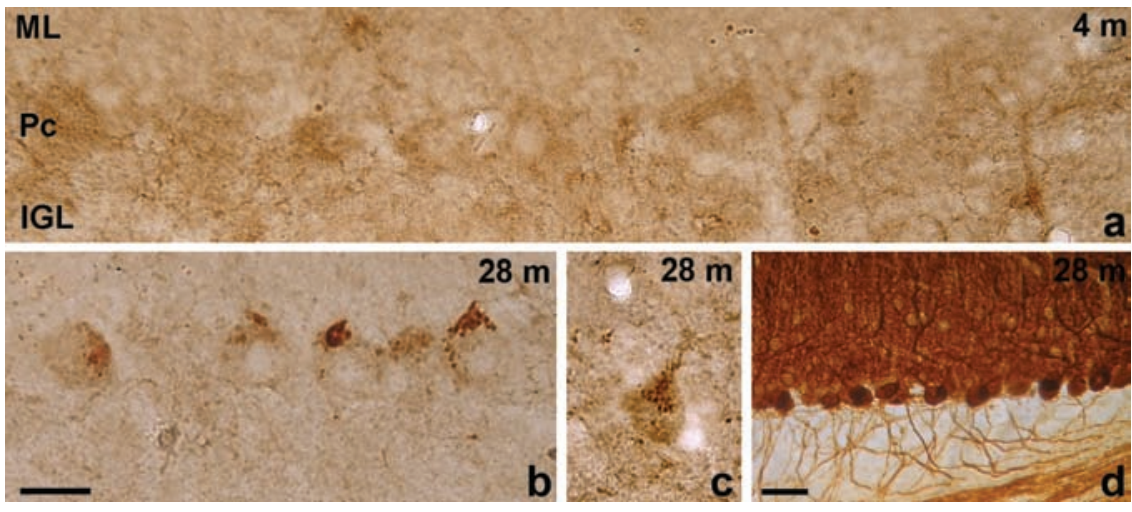

Figure 3. a) In the 4-month-old rat cerebellum, in the most rostral and caudal lobules, no immunoreaction product is present in the Purkinje cell layer; $b, c)$ representative photographs of portions of the most rostral and caudal cerebellar lobules in 28-month-old rats. Punctate immunoreaction product is visible at the apical pole of Purkinje cells; d) particular of the most caudal cerebellar lobules in a section immunostained for calbindin from 28-month-old rat. The cytoarchitecture is apparently well preserved. ML, molecular layer; Pc, Purkinje cell layer; IGL, granule cell layer. Scale bar a,b,c: $20 \mu \mathrm{m}$; d: $50 \mu \mathrm{m}$. 
muscle tone, and that will have an unconfutable answer only when the function of NG2 cells will be fully elucidated.

In aged rats, in lobules I-IV and IX-X, NG2 cells are no more present in the IGL and WM and in the ML they appear paler (Figure 2b) than in young-adult animal. However, punctate immunoreaction product is present in correspondence with the apical pole and stem dendrite of Purkinje cells (Figure 3). The punctate reaction in lobules belonging to the spinocerebellum may represent profiles of hypertrophic NG2 cell processes that ensheath the soma and stem dendrite of Purkinje cells. This is the cell region where climbing fibers from neurons of the inferior olivary complex form the majority of synapses with Purkinje cells. The cerebellum/ inferior olivary complex system plays a major role as a comparator of sensory information and motor output. Climbing fibers inform about errors in the execution of a movement, so that the cerebellum can adjust it. In this view, since climbing fibers establish synapses with NG2 cells, ${ }^{11}$ also NG2 cells could be informed and modulate Purkinje cell activity accordingly. On the other hand, it cannot be excluded that the punctate immunoreaction product represents extracellular product. In fact, the large NG2 proteoglycan ectodomain may be cleaved and released in the extracellular matrix. ${ }^{14}$ This would also explain the pale staining of NG2 cells residing in the ML in 28-month-old animals.

\section{References}

1. Butt AM, Hamilton N, Hubbard P, Pugh M, Ibrahim M. Synantocytes: the fifth element. J Anat 2005;207:695-706.

2. Nishiyama A, Watanabe M, Yang Z, Bu J. Identity, distribution and development of polydendrocytes: NG2-expressing glial cells. J Neurocytol 2002;31:437-55.

3. Reyners H, Gianfelici de Reyners E, Regniers L, Maisin JR. A glial progenitor cell in the cerebral cortex of the adult rat. $\mathrm{J}$ Neurocytol 1986;15:53-61.

4. Levine JM, Reynolds R, Fawcett JW. The oligodendrocyte precursor cell in health and disease. Trends Neurosci 2001;24:39-47.

5. Peters A. A fourth type of neuroglia cell in the adult nervous system. J Neurocytol 2004;33:345-57.

6. Reynolds R, Dawson M, Papadopoulos D, Polite A. Di Bello IC, Pham-Dinh D, et al. The response of NG2-expressing oligodendrocyte progenitors to demyelination in MOG-EAE and MS. J Neurocytol 2002;31: 523-36.

7. Watanabe M, Toyama Y, Nishiyama A. Differentiation of proliferated NG2-positive glial progenitor cells in a remyelinating lesion. J Neurosci Res 2002;69:826-36.

8. Alonso G. NG2 proteoglycan-expressing cells of the adult rat brain: Possible involvement in the formation of glial scar astrocytes following stab wound. Glia 2005;49:318-38.

9. Bergles DE, Roberts JD. Somogyi P, Jar CE. Glutamatergic synapses on oligodendrocyte precursor cells in the hippocampus. Nature 2000;405:187-91.

10. Lin SC, Bergles DE. Synaptic signaling between GABAergic interneurons and oligodendrocyte precursor cells in the hippocampus. Nat Neurosci 2004;7:24-32.

11. Lin SC, Huck JHJ, Roberts JD, Macklin WB, Somogyi P, Bergles DE. Climbing fiber innervations of NG2-expressing glia in the mammalian cerebellum. Neuron 2005; 46 : 773-85.

12. Larsell 0 . The morphogenesis and adult pattern of the lobules and fissures of the cerebellum of the white rat. J Comp Neurol 1952;97:281-356.

13. Paukert M, Bergles DE. Synaptic communication between neurons and NG2+ cells. Curr Open Neurobiol 2006;16:515-21.

14. Sakry D, Karram K, Trotter J. Synapses between NG2 glia and neurons. J Anat 2011; 219:2-7.

15. Seene T, Kaasik P, Riso EM. Review on aging, unloading and reloading: changes in skeletal muscle quantity and quality. Arch Gerontol Geriatr 2012;54:374-80. 